\title{
Effects of Night Sleep on Motor Learning Using Transcranial Magnetic Stimulation
}

\author{
Hae Jin Lee, M.D., Yong Won Park, M.D., Dae Ho Jeong, M.D., Han Young Jung, M.D. \\ Department of Rehabilitation Medicine, Inha University College of Medicine, Incheon 400-711, Korea
}

\begin{abstract}
Objective To investigate the effects of night sleep on motor cortical excitability with TMS (transcranial magnetic stimulation) and finger tapping performance.

Method Eight volunteers were enrolled to investigate the effects of day wake or night sleep on motor learning and finger performance. Each subject underwent a finger tapping task over a 12 hour period, which was employed to evaluate the motor cortical excitability affected by motor learning. Starting at 9:00 am for the day wake cycle and restarting at 9:00 pm for the night sleep cycle. The finger tapping task was the index finger of the non-dominant hand with the Hangul word personal computer (PC) training program. The data was assessed by comparing the changes observed with the cortical excitability and finger tapping performance tests between the day wake and night sleep after equivalent amounts of training.

Results The results showed that in paired-pulse techniques, there was a significant decrease of intracortical inhibition (ICI) in the morning following the night sleep cycle $(\mathrm{p}<0.05)$, but no significant change was seen in the ICI in the evening for the day wake cycle. In addition a significant decrease of the ICI was observed in comparison to the morning following the night sleep cycle and the evening following the day wake cycle $(\mathrm{p}<0.05)$. The $140 \%$ recruitment curve (RC) and accuracy of the finger tapping performance demonstrated a significant improvement for both cycles $(\mathrm{p}<0.05)$.

Conclusion Through this study, we observed that the Hangul typing practice requires both explicit and implicit skill learning. And also the off-line learning during a night of sleep may be affected by an inhibitory neurotransmitter related synaptic plasticity and by the time dependent learning with recruitments of remote or less excitable motor neurons in the primary motor cortex.
\end{abstract}

Key Words Night sleep, Off-line learning, Hangul typing practice, Transcranial magnetic stimulation (TMS)

Received May 16, 2011; Accepted January 11, 2012

Corresponding author: Han Young Jung

Department of Rehabilitation Medicine, Inha University College of Medicine, 7-206, Sinhung-dong-3 ga, Jung-gu, Incheon 400-711, Korea Tel: +82-32-890-2480, Fax: +82-32-890-2486, E-mail: rmjung@inha.ac.kr (c) This is an open-access article distributed under the terms of the Creative Commons Attribution Non-Commercial License (http:// creativecommons.org/licenses/by-nc/3.0) which permits unrestricted noncommercial use, distribution, and reproduction in any medium, provided the original work is properly cited.

Copyright $\odot 2012$ by Korean Academy of Rehabilitation Medicine

\section{INTRODUCTION}

In rehabilitation, improvements in motor learning are achieved through goal directed repetitive exercise. Repetitive exercise itself can increase motor learning, however, according to recent studies, learning also occurs between training sessions and is referred to as off-line learning. ${ }^{1}$ In off-line learning, the process of memory consolidation is important. Post-training consolidation offers a second, delayed stage of learning that develops in the absence of 
further training and it can be affected by many circumstances specifically including the type of motor training, memory and sleep. ${ }^{2-4}$

Walker et al. ${ }^{5}$ presented functional magnetic resonance imaging data which demonstrated alterations in the representation of motor memory after a night of sleep. However, there are no recent studies which present neurophysiological data on the relationship between sleep and motor learning and specifically, cortical motor excitability. Here we focused on presenting neurophysiological data regarding how sleep affects the motor cortical activity using transcranial magnetic stimulation (TMS).

\section{MATERIALS AND METHODS}

\section{Subjects}

Eight subjects with no neurological or psychiatric conditions were recruited in this study. All of the subjects were right-handed, two were female and the remaining six were male subjects with a mean age of 27 (24-36). We restricted any alcohol or drug intake that might affect the participants' sleep cycle during the 24 hours before and after the experiment. We also recommended that the subjects maintain a regular sleep cycle during the experimental period.

\section{Methods}

All subjects underwent a serial reaction time (SRT) finger tapping task and TMS comparison study with an interval of $12 \mathrm{hrs}$. For the day wake cycle, the baseline study was performed at 9:00 am and the comparison study was initiated at 9:00 pm. For the night sleep cycle, the base-
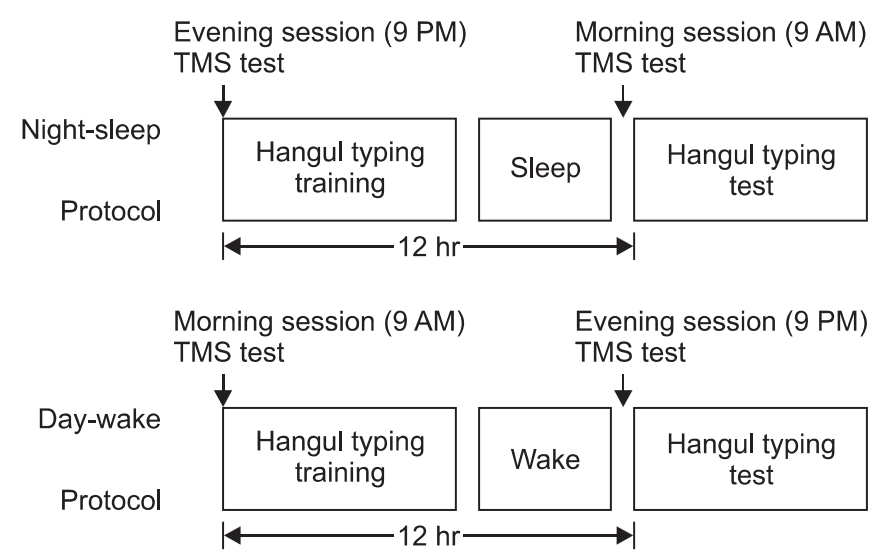

Fig. 1. The Hangul typing practice and test protocols, composed of a night sleep cycle and a day wake cycle. line study was performed at 9:00 pm and the comparison study was done at 9:00 am. Between each cycle, there was an interval of $48 \mathrm{hrs}$, and all of the subjects were encouraged to sleep for more than eight hours (Fig. 1).

The SRT is a commonly used method for the measurement of implicit learning. In the SRT task, subjects are asked to respond to a fixed set of cues. The reaction time to the cues was measured. Learning is defined as increasingly faster response times.

In this research, the finger tapping task was implemented using the index finger of the non-dominant hand in a seated position on a chair while maintaining a fixed distance from the subjects' eye and the PC monitor. Typing was completed with the same PC word training program (Hangul 2007 word training program step 1: typing the middle line of the key board upon visual cues) repeating the task for 10 times and checking both the time and accuracy of the performance level. ${ }^{6}$ During the finger tapping program the subjects' wrist was immobilized with a resting splint to encourage the movement of the first dorsal interossei muscle.

Along with the task performance level test, the cortical motor excitability was tested using transcranial magnetic stimulation (TMS) with the MAGSTIM 200 Mono Pulse (Magstim Co., Whiteland Dyfed, UK). Using a figure-8 electromagnetic coil, we stimulated the motor cortex that is involved in the activation of the first dorsal interossei of the non-dominant hand. The axonal membrane excitability of the corticocortical axon is measured using the resting motor threshold (rMT), and the corticocortical excitability is measured using the testing recruitment curve (RC). ${ }^{7}$ The synaptic excitability of the inhibitory and excitatory circuit at the level of the motor cortex is tested using paired-pulse measurement of the intracortical inhibition and intracortical facilitation (ICI \& ICF). ${ }^{8}$

The rMT is the minimal stimulation intensity in which the motor potential larger than $50 \mu \mathrm{V}$ is evoked more than five times throughout ten consecutive stimulations. $\mathrm{RC}$ is the mean amplitude (stimulated ten times, peak to peak; $\mu \mathrm{V}$ ) of the motor evoked potential (MEP) stimulated at $120 \%$ and $140 \%$ of the rMT. ${ }^{9}$

The Conditioning stimulation of the paired-pulse stimulation is done at $80 \%$ of the rMT and test stimulation was performed at $130 \%$ of the rMT. The inter-stimulus interval (ISI) of the ICI was set at $3 \mathrm{msec}$ and the ISI of the ICF was set at 13 msec. ${ }^{8}$ The mean MEP amplitudes in each ICI and ICF were expressed as the percent of the 
mean single pulse test MEP amplitude.

The data was assessed in the same subjects after an initial training session by comparing the differences in the cortical excitability and hand taping performance during the 12 hours wakefulness compared to results obtained during the 12 hours of wake following a subsequent night's sleep with repeated measurements including the analysis of variance (repeated measure ANOVA). In addition, we assessed the presence of time dependent learning by comparing the differences between two pretraining conditions (AM9 of day wake cycle and PM9 of night sleep cycle) and also the differences between two post-training states (PM9 of day wake cycle and AM9 of night sleep cycle) with the Mann-Whitney U test. Our data had a normal distribution which was analyzed by the Shapiro-Wilk test. p-values lower than 0.05 were assumed to be statistically significant results.

\section{RESULTS}

The mean value of the rMT in the day wake cycle was $51.00 \pm 9.89 \%$ at 9 am and $53.25 \pm 11.15 \%$ for the 9 pm cycle. In the night sleep cycle, the mean value at 9 pm was $50.50 \pm 8.43 \%$ and the mean value at 9 am was $49.50 \pm 9.70 \%$, however, there were no statistically significant differences between the cycles ( $p>0.05$ ) (Fig. 2).

The mean amplitude of the MEP stimulated at $120 \%$ of the rMT for the day wake cycle demonstrated a reading of $783.50 \pm 632.07 \mu \mathrm{V}$ at 9 am and $721.25 \pm 496.45 \mu \mathrm{V}$ for the $9 \mathrm{pm}$ cycle. In the night sleep cycle, the mean ampli-

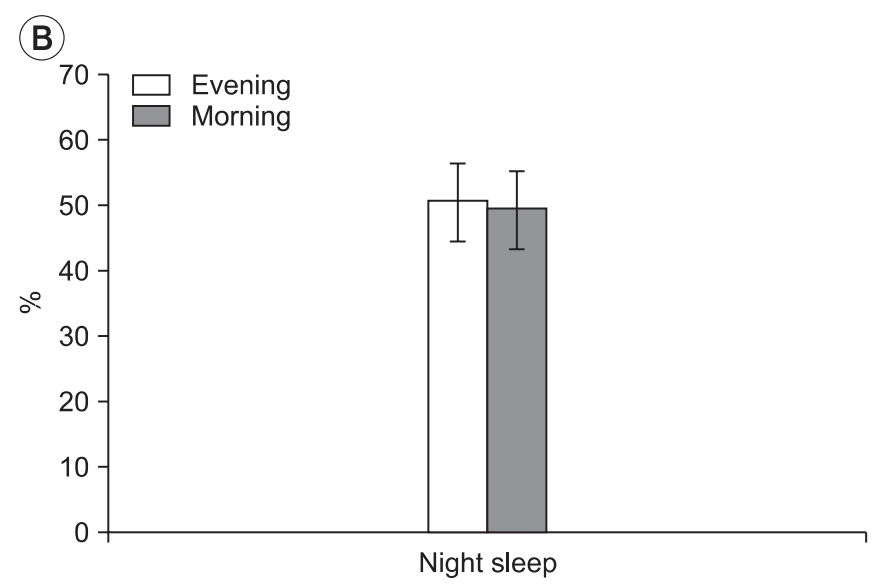

(A)

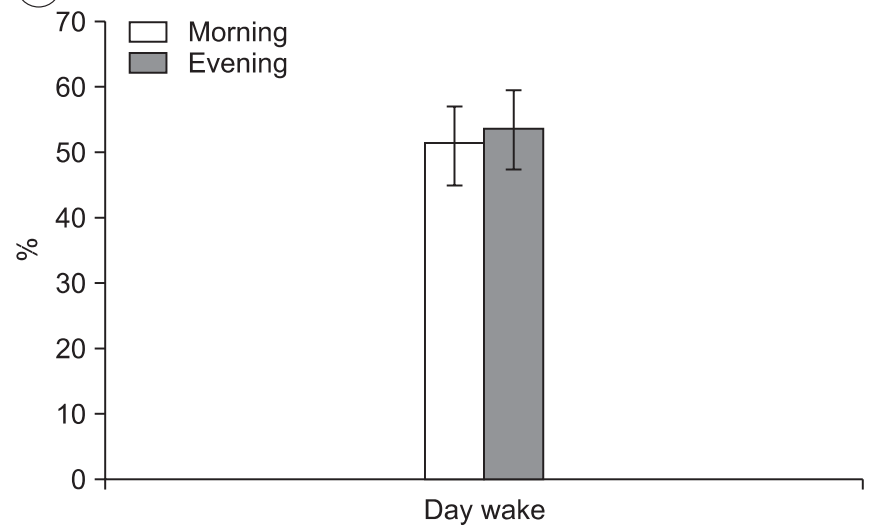

Fig. 2. Changes in the resting motor threshold in the day wake (A) and night sleep (B) cycle. Values are the means \pm standard deviation and analyzed by repeated measure ANOVA $(\mathrm{p}>0.05)$.

(A)

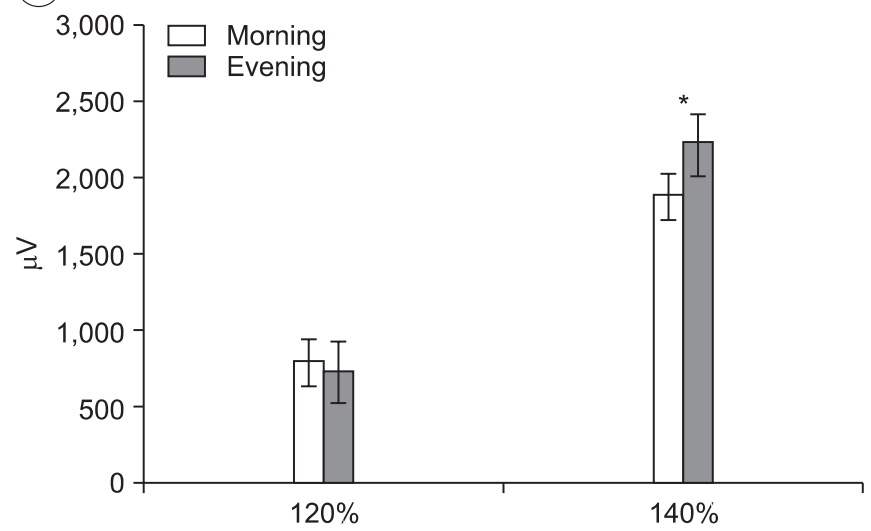

(B)

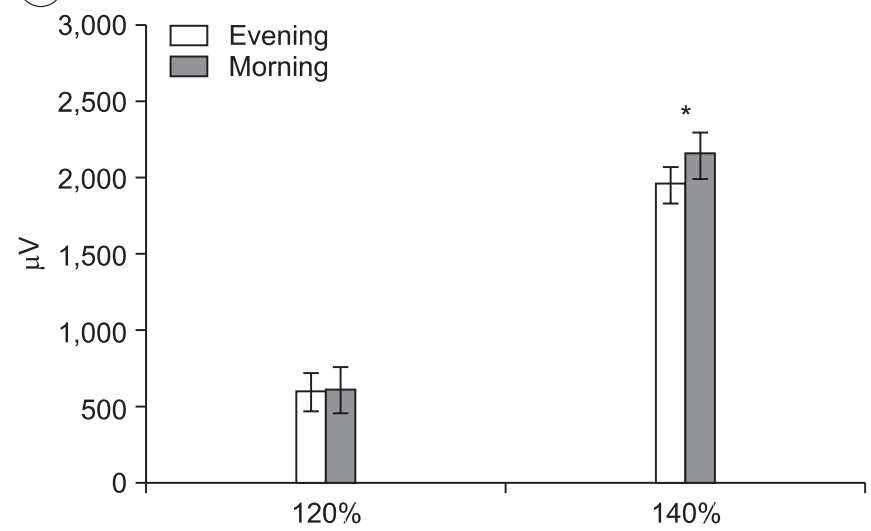

Fig. 3. Changes in the single pulse TMS, recruitment curves stimulated by $120 \%$ and $140 \%$ of the resting motor threshold in the day wake (A) and the night sleep (B) cycle. Values are the means \pm standard deviation and analyzed by repeated measure ANOVA $\left({ }^{*} \mathrm{p}<0.05\right)$. 
tude was $595.75 \pm 559.80 \mu \mathrm{V}$ at $9 \mathrm{pm}$ and $606.25 \pm 429.38$ $\mu \mathrm{V}$ at 9 am after more than eight hours of sleep. All mean amplitudes of these MEPs for both the day wake and the night sleep cycle also showed no statistically meaningful changes ( $p>0.05$ ) (Fig. 3).

The mean amplitude of the MEP stimulated at $140 \%$ of the rMT for the day wake cycle showed 1,879.75 \pm 947.16 $\mu \mathrm{V}$ at 9 am and $2,216.00 \pm 827.22 \mu \mathrm{V}$ for the $9 \mathrm{pm}$ cycle. In the night sleep cycle, the mean amplitude was $1,951.50 \pm 373.89 \mu \mathrm{V}$ at $9 \mathrm{pm}$ and $2,147.25 \pm 469.51 \mu \mathrm{V}$ at 9 am after more than eight hours of sleep. In both the day wake and night sleep cycles the mean amplitudes of MEPs stimulated at $140 \%$ of the rMT showed significant increase at the interval of 12 hours ( $p<0.05)$ (Fig. 3).

In the paired pulse stimulation, the mean value of the ICI for the night sleep cycle was $-36.43 \pm 17.14 \%$ at

(A)

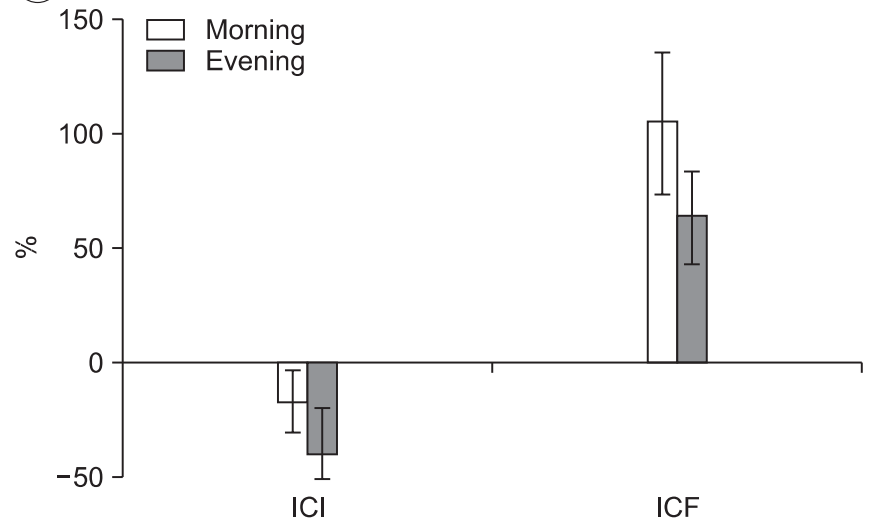

$9 \mathrm{pm}$ and $-16.33 \pm 22.13 \%$ for the 9 am cycle after more than eight hours of sleep $(\mathrm{p}<0.05)$. For the day wake cycle, the mean value was $-16.42 \pm 54.68 \%$ for 9 am and $-39.54 \pm 17.01 \%$ for the $9 \mathrm{pm}$ cycle. Unlike the night sleep cycle, this value is statistically insignificant for the day wake cycle ( $p>0.05$ ) (Fig. 4).

Continuing with the paired pulse stimulation, the mean value of the ICF in the day wake cycle was $104.54 \pm 65.51 \%$ at 9 am and $63.45 \pm 61.20 \%$ for the 9 pm cycle. For the night sleep cycle, the mean value was $85.34 \pm 49.27 \%$ at 9 pm and $109.48 \pm 62.53 \%$ for the 9am cycle which demonstrated an interval increase, but neither the day wake nor the night sleep cycle showed statistically significant data (p>0.05) (Fig. 4).

The mean value of the performance speed during the finger tapping task was $123.75 \pm 1.26 \mathrm{sec}$ at 9 am and

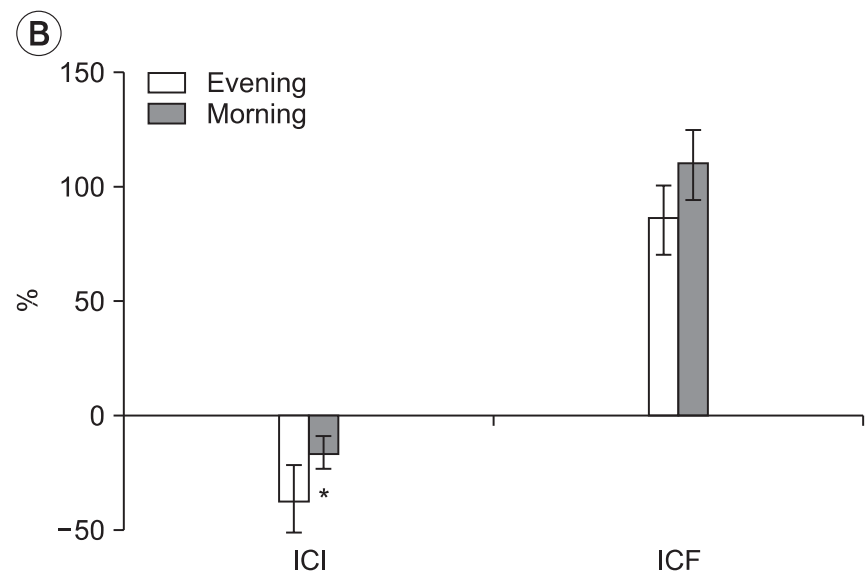

Fig. 4. Changes in the ICI and ICF in the day wake (A) and night sleep (B) cycle. Values are the means \pm standard deviation and analyzed by repeated measure ANOVA $\left({ }^{*} \mathrm{p}<0.05\right)$.

(A)

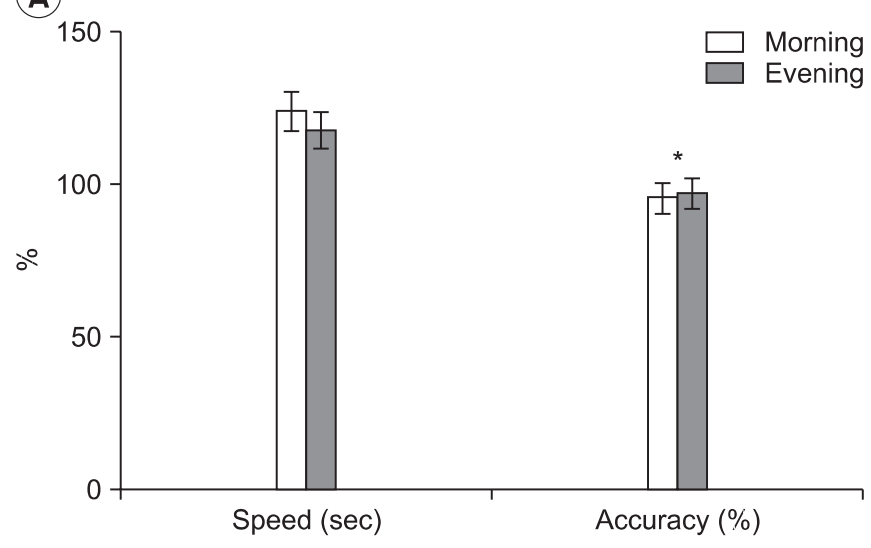

(B)

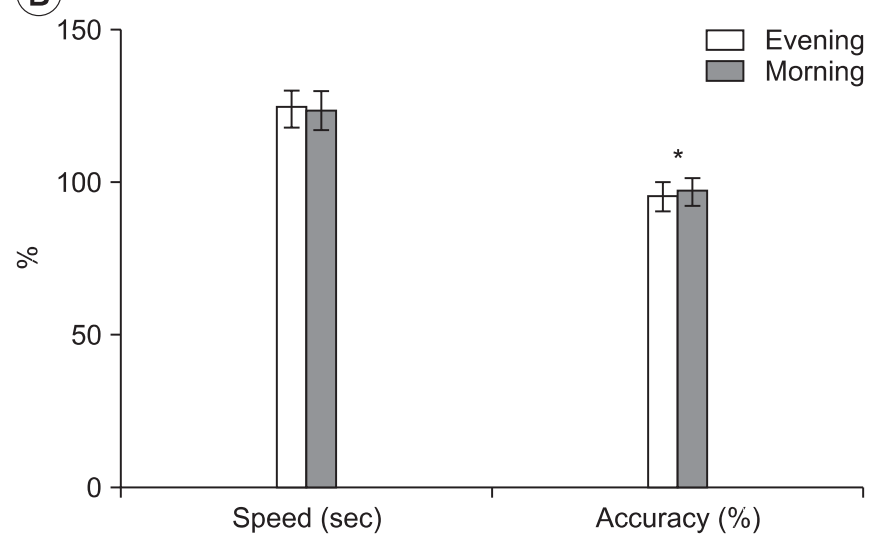

Fig. 5. Changes in the speed and accuracy in the day wake (A) and the night sleep (B) cycle. Values are the means \pm standard deviation and analyzed by repeated measure ANOVA $\left({ }^{*} \mathrm{p}<0.05\right)$. 
$117.60 \pm 13.34 \mathrm{sec}$ at $9 \mathrm{pm}$ for the day wake cycle, and $123.85 \pm 0.89 \mathrm{sec}$ at $9 \mathrm{pm}$ and $123.25 \pm 2.63 \mathrm{sec}$ at 9 am for the night sleep cycle, respectively. These values of performance speed showed a tendency to decrease for both cycles but were statistically insignificant ( $p>0.05$ ) (Fig. 5).

The mean value of performance accuracy in the finger tapping task was $95.45 \pm 1.38 \%$ at 9 am and $96.95 \pm 0.55 \%$ at $9 \mathrm{pm}$ for the day wake cycle, and $95.10 \pm 1.49 \%$ at $9 \mathrm{pm}$ and $96.70 \pm 0.68 \%$ at 9 am for the night sleep cycle, respectively. These performance accuracy tests showed a statistically significant increase for both cycles ( $\mathrm{p}<0.05)$ (Fig. 5).

In assessing the presence of time dependent learning through the comparison of differences between the two pre-training conditions and the two post-training states, the mean value of the ICI exhibited significant differences between the post-training states which were in the evening of the day wake cycle and in the morning of the night sleep cycle $(\mathrm{p}<0.05)$. There were no significant differences observed in the others including rMT, ICF, RC120\%, and RC140\% (p>0.05) (Table 1).

\section{DISCUSSION}

The changes occurring in between practice sessions and

Table 1. Comparison of the TMS Parameters between the Day Wake and Night Sleep Cycles

\begin{tabular}{lcc}
\hline & $\begin{array}{c}\text { Morning in } \\
\text { day wake }\end{array}$ & $\begin{array}{c}\text { Evening in } \\
\text { night sleep }\end{array}$ \\
\hline $\mathrm{rMT}(\%)^{*}$ & $51.00 \pm 9.89$ & $50.50 \pm 8.43$ \\
$\mathrm{RC} 120(\mu \mathrm{V})$ & $783.50 \pm 632.07$ & $595.75 \pm 559.80$ \\
$\mathrm{RC} 140(\mu \mathrm{V})$ & $1,879.75 \pm 947.16$ & $1,951.50 \pm 373.89$ \\
$\mathrm{ICI}(\%)^{\dagger}$ & $-16.42 \pm 54.68$ & $-36.43 \pm 17.14$ \\
$\mathrm{ICF}(\%)^{\dagger}$ & $104.54 \pm 65.51$ & $85.34 \pm 49.27$ \\
\hline & Evening in & Morning in \\
& day wake & night sleep \\
\hline $\mathrm{rMT}(\%)^{*}$ & $53.25 \pm 11.15$ & $49.50 \pm 9.70$ \\
\hline $\mathrm{RC} 120(\mu \mathrm{V})$ & $721.25 \pm 496.45$ & $606.25 \pm 429.38$ \\
$\mathrm{RC} 140(\mu \mathrm{V})$ & $2,216.00 \pm 827.22$ & $2,147.25 \pm 469.51$ \\
$\mathrm{ICI}(\%)^{\dagger}$ & $-39.54 \pm 17.01$ & $-16.33 \pm 22.13^{\dagger}$ \\
$\mathrm{ICF}(\%)^{\dagger}$ & $63.45 \pm 61.20$ & $109.48 \pm 62.53$ \\
\hline
\end{tabular}

rMT: Resting motor threshold, RC: Recruitment curve, ICI: Intracranial inhibition, ICF: Intracortical facilitation *\% of the maximum stimulator output, ${ }^{\dagger} \%$ of the mean single pulse test MEP amplitude, Values are means+standard deviation and analyzed by the MannWhitney U test $\left({ }^{\ddagger} \mathrm{p}<0.05\right)$ without physical practice are defined as off-line learning. Off-line learning is affected by several factors including, the type of memory, practice, and sleep as well as others. Recently there has been increasing interest in the effect of sleep on motor learning and also off-line learning. ${ }^{2-4}$

In several studies, motor performance of the finger task has shown $20-33 \%$ improvement after eight hours of a night sleep. ${ }^{10,11}$ Overnight improvements are observed in the SRT to finger tapping in patients with a prefrontal cortex infarction, ${ }^{12}$ and the error rate of moving objects using fingers in patients with middle cerebral artery infarction decreased by $12-14 \%$ after a full night of sleep.

Walker et al. ${ }^{5}$ used functional magnetic resonance imaging to determine whether a sleep cycle taken after practice has an effect on off-line learning. Following a night of sleep relative to wakefulness, regions of increased activation were expressed in the right primary motor cortex, medial prefrontal lobe, hippocampus and left cerebellum. These changes are related to precise motor performance and accuracy achieved during the finger task activities.

In this study, we determined the neurophysiological effect of night sleep on the motor cortical excitability using TMS. The process of acquiring a motor skill, or practice of a motor task are to be associated with alterations in cortical motor excitability, which may be a result of motor learning. ${ }^{13}$ Also off-line consolidation involves the strengthening of memory representations at the synaptic level, ${ }^{14}$ which can be expressed by changes in the ICI or ICF.

In our study, the ICI in paired pulse stimulation demonstrated a decrease in the night sleep cycle which suggests that sleep has an effect on motor learning through the disinhibition of the inhibitory neurotransmitter, GABA (Gamma Amino Butyric Acid) related neurotransmitters as opposed to changes in the neuronal membrane stability.

In the latest studies, not all types of motor learning are affected by off-line learning. The types of motor learning that can be conducted unintentionally are not affected by sleep, but motor learning that requires intentional recall of memory show vast improvements following sleep. ${ }^{3,4}$ However, the time dependent motor learning is acquired by the unintentional procedural learning task and also the magnitude of day time and overnight improvements are similar. $^{1}$

According to the studies regarding off-line learning and 
the type of memory, motor learning that utilizes explicit memories is much more affected by sleep than motor learning that utilizes implicit or procedural memory. ${ }^{15}$ In other words, there would be more improvement during non practicing periods if the individual who is learning the motor skill intentionally recalls the memories during practice.

TMS has been used to investigate the mechanism of various interventions or drugs affecting cortical excitability. ${ }^{16,17}$ The MT in the resting muscle reflects the excitability of the central core of neurons, depending on the excitability of the individual neurons and their local density. The ICI and ICF are assessed by paired-pulse TMS and reflect the synaptic activity in the cortex. At below 5 milliseconds, there is inhibition, whereas there is facilitation during intervals between 8 and 30 milliseconds. ${ }^{8,9}$ The ICI is likely largely a GABAergic effect, ${ }^{8}$ whereas the ICF is largely a glutamatergic effect. ${ }^{18}$ The RC is the growth of the MEP size as a function of stimulus intensity. This measurement is partially understood, but appears to involve neurons in addition to less excitable or spatially remote neurons. ${ }^{19}$

In our data, the fact that the rMT and ICF did not change during the day wake and night sleep cycle suggests no involvement of the neuronal cell membrane excitability, facilitatory mechanisms in time dependent learning, or the off-line learning after the Hangul typing practice. However, the primary motor cortex could have a critical contribution to off line learning overnight through the disinhibition of the ICI. It has been confirmed that the day time improvement after practice was blocked by repetitive TMS on the primary motor cortex, but the improvement observed following the night sleep cycle was not blocked. ${ }^{1}$ In addition, through our comparison study on the TMS parameters between the day wake and night sleep cycle, the disinhibition of the ICI was advanced by off-line learning via night sleep, but did not progress as a result of practice dependent learning.

The type of memory needed to carry out the Hangul word training program used in this study is procedural meaning that many people already know the keyboard sequence. However, the sequence is given in random order so there is a portion of explicit memory. That improvement in accuracy of the Hangul word typing following practice for both the day wake and night sleep cycle suggest the involvement of unintentional characteristics of motor learning in Hangul word typing, a result of both explicit and implicit learning. This fact also leads to an increase in the mean amplitude of the MEP stimulated at $140 \%$ of the rMT for both the day wake and night sleep cycle. The same phenomenon was observed in individuals that study dance and the reliance on intentional instruction regarding the sequence of dance steps apart from unintentional repetitive practice. ${ }^{20}$

These sleep induced off-line learning effects are not only present in normal healthy adults, but also present in stroke patients. ${ }^{21}$ However, neurophysiological studies involving this particular field are not sufficient. In the future, further studies addressing the relationship between sleep and motor learning in stroke patients might be an important examination.

\section{CONCLUSION}

Even though practice induced motor learning is one of the most important methods in rehabilitation therapy, off-line learning which is defined as learning without practice could also contribute to the achievement of skills.

In our study, the Hangul word practice, which is involved in both explicit and implicit leaning, has off line learning effects and also day time learning effects. These mixed types, intentional and unintentional practices, might have not only have an effect in off line learning by night sleep but also time dependent learning throughout the day time. Off-line learning with intentional motor practice was developed by disinhibition of the GABA related inhibitory neurotransmitter(s) and time dependent learning may be achieved by neuronal recruitment of remote or less excitable motor neurons in the primary motor cortex.

\section{ACKNOWLEDGEMENTS}

This work was supported by the Inha University Research Grant.

\section{REFERENCES}

1. Robertson EM, Press DZ, Pascual-Leone A. Off-line learning and the primary motor cortex. J Neurosci 2005; 25: 6372-6378

2. Robertson EM, Pascual-Leone A, Miall RC. Current concepts in procedural consolidation. Nat Rev 
Neruosci 2004; 5: 576-582

3. Robertson EM, Pascual-Leone A, Press DZ. Awareness modifies the skill-learning benefits of sleep. Curr Biol 2004; 14: 208-212

4. Diekelmann S, Born J. The memory function of sleep. Nat Rev Neruosci 2010; 11: 114-126

5. Walker MP, Stickgold R, Alsop D, Gaab N, Schlaug G. Sleep-dependent motor memory plasticity in the human brain. Neuroscience 2005; 133: 911-917

6. Roberson EM. The serial reaction time task: implicit motor skill learning? J Neurosci 2007; 27: 10073-10075

7. Devanne H, Lavoie BA, Capaday C. Input-output properties and gain changes in the human corticospinal pathway. Exp Brain Res 1997; 114: 329-338

8. Ziemann U, Rothwell JC, Ridding MC. Interaction between intracortical inhibition and facilitation in human motor cortex. J Physiol 1996; 496: 873-881

9. Kujirai T, Caramia MD, Rothwell JC, Day BL, Thompson PD, Ferert A, Wroe S, Asselman P, Marsden CD. Corticocortical inhibition in human motor cortex. J Physiol 1993; 471: 501-519

10. Steriade M, Timofeev I. Neuronal plasticity in thalamocortical networks during sleep and waking oscillations. Neuron 2003; 37: 563-576

11. Walker MP, Brakefield T, Morgan A, Hobson JA, Stickgold R. Practice with sleep makes perfect: sleepdependent motor skill learning. Neuron 2002; 35: 205211

12. Karni A, Meyer G, Jezzard P, Adams MM, Turner R, Ungerleider LG. Functional MRI evidence for adult motor cortex plasticity during motor skill learning.
Nature 1995; 14: 155-158

13. Perez MA, Lungholt BK, Nyborg K, Nielsen JB. Motor skill training induces changes in the excitability of the leg cortical area in healthy humans. Exp Brain Res 2004; 159: 197-205

14. Dudai Y. The neurobiology of consolidations, or, how stable is the engram? Annu Rev Psychol 2004; 55: 5186

15. Smith C. Sleep states and memory processes in humans: procedural versus declarative memory systems. Sleep Med Rev 2001; 5: 491-506

16. Hallett M. Transcranial magnetic stimulation: a useful tool for clinical neurophysiology. Ann Neurol 1996; 40: 344-345

17. Ziemann U, Lonnecker S, Steinhoff BJ, Paulus W. Effects of antiepileptic drugs on motor cortex excitability in humans: a transcranial magnetic stimulation study. Ann Neurol 1996; 40: 367-378

18. Liepert J, Schwenkreis P, Tegenthoff M, Malin JP. The glutamate antagonist riluzole suppresses intracortical facilitation. J Neural Transm 1997; 104: 1207-1214

19. Ridding MC, Rothwell JC. Stimulus/response curves as a method of measuring motor cortical excitability in man. Electroencephalogr Clin Neurophysiol 1997; 105: 340-344

20. Diekelmann S, Wilhelm I, Born J. The whats and whens of sleep-dependent memory consolidation. Sleep Med Rev 2009; 13: 309-321

21. Siengsukon CF, Boyd LA. Sleep to learn after stroke: implicit and explicit off-line motor learning. Neurosci Lett 2009; 451: 1-5 